\title{
DERIVATIONS WHICH ARE INNER AS COMPLETELY BOUNDED MAPS
}

\author{
ILJA GOGIĆ
}

\begin{abstract}
We consider derivations in the image of the canonical contraction $\theta_{A}$ from the Haagerup tensor product of a $C^{*}$-algebra $A$ with itself to the space of completely bounded maps on $A$. We show that such derivations are necessarily inner if $A$ is prime or if $A$ is central. We also provide an example of a $C^{*}$-algebra which has an outer derivation implemented by an elementary operator.
\end{abstract}

Mathematics subject classification (2010): 46L05, 46L07, 47B47.

Keywords and phrases: $C^{*}$-algebra, ideals, quasicentral, central, derivation, completely bounded map.

\section{REFERENCES}

[1] C. A. Akemann, G. A. Elliot, G. K. Pedersen, and J. Tomiyama, Derivations and multipliers of $C^{*}$-algebras, Amer. J. Math., 98 (1976), 679-708.

[2] S. D. Allen, A. M. Sinclair And R. R. Smith, The ideal structure of the Haagerup tensor product of $C^{*}$-algebras, J. reine angew. Math., 442 (1993), 111-148.

[3] P. ARa And M. Mathieu, Local Multipliers of $C^{*}$-algebras, Springer, London, 2003.

[4] R. J. ARChBold, Density theorems for the centre of a $C^{*}$-algebra, J. London Math. Soc., 2, 10 (1975), 189-197.

[5] R. J. Archbold, Topologies for Primal Ideals, J. London Math. Soc., 2, 36 (1987), 524-542.

[6] R. J. Archbold And D. W. B. Somerset, Quasi-standard $C^{*}$-algebras, Math. Proc. Cambridge Philos. Soc, 107 (1990), 349-360.

[7] R. J. Archbold, D. W. B. Somerset And R. M. Timoney, Completely bounded mappings and simplicial complex structure in the primitive ideal space of a $C^{*}$-algebra, Trans. Amer. Math. Soc., 361 (2009), 1397-1427.

[8] R. J. Archbold, D. W. B. Somerset And R. M. Timoney, On the central Haagerup tensor product and completely bounded mappings of a $C^{*}$-algebra, J. Funct. Anal., 226 (2005), 406-428.

[9] B. BLACKADAR, Operator Algebras, Encyclopaedia of Mathematical Sciences 122, Springer-Verlag, Berlin, 2006.

[10] D. P. Blecher And C. Le Merdy, Operator algebras and Their modules, Clarendon Press, Oxford, 2004.

[11] C. Delaroche, Sur les centres des $C^{*}$-algèbres, Bull. Sc. Math., 91 (1967), 105-112.

[12] C. Delaroche, Sur les centres des $C^{*}$-algèbres II, Bull. Sc. Math., 92 (1968), 111-128.

[13] J. DiXMiER, $C^{*}$-algebras, North-Holland, Amsterdam, 1977.

[14] J. M. G. FELL, The structure of algebras of operator fields, Acta Math., 106 (1961), 233-280.

[15] I. KAPLANS KY, Normed algebras, Duke Math. J., 16 (1949), 399-418.

[16] B. Magajna, Uniform approximation by elementary operators, Proc. Edinburgh Math. Soc., 52 (2009), 731-749.

[17] V. PAulsen, Completely Bounded Maps and Operator Algebras, Cambridge University Press, 2003.

[18] G. K. Pedersen, $C^{*}$-algebras and their automorphism groups, Academic Press, London, 1979.

[19] I. Raeburn And D. P. Williams, Morita Equivalence and Continuous-Trace C* -Algebras, Mathematical Surveys and Monographs 60, Amer. Math. Soc., Providence, RI, 1998.

[20] S. SAKAI, $C^{*}$-algebras and $W^{*}$-algebras, Springer-Verlag, 1971.

[21] R. R. Sмith, Completely Bounded Maps and the Haagerup Tensor Product, J. Functional Analysis, 102 (1991), 156-175. 
[22] D. W. Somerset, The central Haagerup tensor product of a $C^{*}$-algebra, J. Operator Theory, 39 (1998), 113-121.

[23] D. W. SOMERSET, The inner derivations and the primitive ideal space of a $C^{*}$-algebra, J. Operator Theory, 29 (1993), 307-321. 